\title{
Prevalence and antimicrobial susceptibility pattern of Citrobacter species in various clinical samples in a tertiary care hospital \\ Poonam $\mathrm{AR}^{1, *}$, Anil Kumar Bilolikar ${ }^{1}$, and Sukrutha Gopal Reddy ${ }^{1}$ \\ ${ }^{1}$ Department of Microbiology, Krishna Institute of Medical Sciences, Minister Road, Secunderabad-500003, Telangana, India
}

\begin{abstract}
Background: Infections with Citrobacter species have been increasing across worldwide. Earlier, considered to be organism of low virulence but now known to cause variety of infections with high morbidity and mortaliy.
\end{abstract}

Objective: The present study aims to determine the prevalence and antibiotic susceptibility pattern of Citrobacter species from various clinical samples in a tertiary care hospital.

Material and methods: This one year prospective observational study, conducted in the Department of Microbiology at Krishna Institute of Medical Sciences, Secunderabad from January 2018 to December 2018. The samples received to the laboratory from both outpatient and inpatient from various departments. Identification and antimicrobial susceptibility of the isolates were done by using Vitek 2 compact automated system (bioMerieux).

Results: In 3,337 culture positive sample, 83 (2.4\%) isolates were found to be culture positive for Citrobacter species where $C$. koseri $(\mathrm{n}=57)$ is predominant followed by $C$. freundii $(\mathrm{n}=24)$ and $C$. amalonaticus $(\mathrm{n}=2)$. Majority of Citrobacter species were isolated from urine samples $56 \%$ followed by pus $21 \%$, sputum $8.43 \%$, blood $7.2 \%$ and in bile fluid 3.6\%. The highest incidence was found in elderly patients of age group 51-80 year age group, more common in males (70\%) than females (30\%).

Conclusion: The present study highlight that Citrobacter species are an emerging pathogen with prevalence of $2.4 \%$. It is the most common uropathogen followed by pus and sputum, and bacteraemia also. C. freundii was comparatively less susceptible as compared to C. koseri. Antibiotic policy should be implemented strictly and followed strongly so that emergence of such low virulent organism with emerging resistance can be controlled.

Keywords: Citrobacter spp.; antimicrobial; susceptibility; Citrobacter koseri; Citrobacter fruendii; Citrobacter amalonaticus; infections; Vitek 2

\footnotetext{
*Corresponding author: Dr. A. R. Poonam, DNB resident, Department of Microbiology, Krishna Institute of Medical Sciences, Minister Road, Secunderabad-500003, Telangana, India. Email: doc.poonam@yahoo.com
}

Received 15 July 2019; Revised 31 August 2019; Accepted 10 September 2019; Published 21 September 2019

Citation: Poonam AR, Bilolikar AK, Reddy SG. Prevalence and antimicrobial susceptibility pattern of Citrobacter species in various clinical samples in a tertiary care hospital. J Med Sci Res. 2019; 7(4):103-108. DOI:

http://dx.doi.org/10.17727/JMSR.2019/7-18

Copyright: (C) 2019 Poonam AR et al. Published by KIMS Foundation and Research Center. This is an open-access article distributed under the terms of the Creative Commons Attribution License, which permits unrestricted use, distribution, and reproduction in any medium, provided the original author and source are credited. 


\section{Introduction}

Infections with Citrobacter species have been increasing across worldwide [1]. Citrobacter species belong to a group of facultative anerobic, gram-negative bacilli belongs to the family Enterobacteriaceae, tribe Citrobactereae [2]. It is found singly or in pairs, and are motile by peritrichous flagella which typically utilize citrate as sole source of carbon [3]. In the environment, they are frequently found in water, soil, food, and as an occasional colonizers of the intestines of human and animals. These organisms are isolated from variety of clinical specimens like urine, pus, blood, and cerebrospinal fluid [4].

There are 11 different species (Citrobacter freundii, Citrobacter koseri, Citrobacter amalonaticus, Citrobacter farmeri, Citrobacter youngae, Citrobacter braakii, Citrobacter werkmanii, Citrobacter sedlakii, Citrobacter rodentium, Citrobacter gillenii, and Citrobacter murliniae) among which the most commonly isolated from human clinical specimens are $C$. koseri (formerly named $C$. diversus), C. freundii, C. youngae, C. braakii, and C. amalonaticus [5].

Earlier, they are recognised as environmental contaminants or colonizers with low virulence, they are now known to cause variety of infections such as the urinary tract, wounds, soft tissue, respiratory tract, liver, bloodstream, biliary tract, peritoneum, meningitis and brain abscess [6].

Although Citrobacter infections occur in adults with immunocompromised state but it is now an emerging cause of UTIs ,neonatal septicaemia, neonatal meningitis, brain abscess with high mortality as high approximately one-third of infants with abscesses die, and one-half sustain CNS damage $[7,8]$.

Citrobacter infections were found to represent $0.8 \%$ of Gram-negative infections in a large surveillance study conducted in American medical centres [9], while, in hospital settings, accounted for $3-6 \%$ of all isolates of Enterobacteriaceae [9]. There are reports of Citrobacter species being a major nosocomial pathogen with multi drug resistant antimicrobial susceptibility [10]. The present study was taken up to determine the prevalence and antibiotic susceptibility pattern of Citrobacter species from various clinical samples in a tertiary care hospital.

\section{Material and methods}

This was a prospective observational study, conducted in the Department of Microbiology at Krishna Institute of Medical Sciences, Secunderabad from January 2018 to December 2018. A total of 17,544 samples received to the laboratory from both outpatient and inpatient from various departments. Out of total samples, 3,337 were culture positive. The samples included urine (clean catch \& catheter catch), pus, and sputum, endotracheal tube secretions, sterile body fluids and blood. All the samples were received in the microbiology laboratory under sterile conditions and were processed as per standard microbiological guidelines.

Specimens were processed on blood agar (BA), Mac Conkey agar (MA) plate and urine samples were processed on cysteine lactose electrolyte deficient (CLED) agar. The inoculated culture plates were incubated at $37^{\circ} \mathrm{C}$ for $16-18$ hours under aerobic conditions. In case of blood sample, paired samples were collected and they were inoculated in BacT Alert bottles which were incubated in BacT Alert 3D (bioMerieux).

After culture growth, bacterial identification and susceptibility was done by VITEK 2 compact (bioMerieux) automated system. Identification of the isolates were done by using Vitek GN-ID card and antimicrobial susceptibility testing was done by using GN AST 280 card.

Antimicrobials used in the VITEK AST 280 card was ampicillin, amoxycillin-clavulanic acid, piperacillintazobactum (TZP), cefuroxime (CXM), ceftriaxone, cefeperazone-sulbactum (SFP), cefepime (FPM), ertapenem (ETP), imipenem, meropenem (MEM), amikacin, gentamycin (GM), nalidixic acid (NA), ciprofloxacin (CIP), tigecycline (TGC), nitrofurantoin (FT), colistin (CS) and cotrimoxazole (SXT). Reporting of susceptibility, intermediate and resistance based on minimum inhibitory concentration values (MIC) was done as per Clinical Laboratory Standard Institute (CLSI) guidelines 2018 [11].

\section{Results}

In 3,337 culture positive sample, 83 (2.4\%) isolates were found to be culture positive for Citrobacter 
species where C. koseri $(\mathrm{n}=57)$ is predominant followed by $C$. freundii $(\mathrm{n}=24)$ and $C$. amalonaticus $(\mathrm{n}=2)$. Majority of Citrobacter species were isolated from urine samples $56 \%(n=47)$ in which clean catch is $(n=37) 44 \%$ is high as compared to catheter catch $(\mathrm{n}=10)$ is $12 \%$ followed by pus $(\mathrm{n}=18) 21 \%$, sputum $(\mathrm{n}=7) \mathrm{8.43 \%}$, blood $(\mathrm{n}=6) 7.2 \%$ and in bile fluid $(n=3)$ is $3.6 \%$ as shown in Table 1 .

Table 1: Distribution of Citrobacter species in various clinical samples.

\begin{tabular}{|c|c|c|c|c|c|}
\hline Sample type & Total & Percentage & C. koseri & C. freundii & C. amalonaticus \\
\hline Urine(Clean catch) & 37 & $44.5 \%$ & 28 & 9 & 0 \\
\hline Urine (Catheter catch) & 10 & $12 \%$ & 7 & 2 & 1 \\
\hline Pus & 18 & $21.6 \%$ & 13 & 4 & 1 \\
\hline Sputum & 7 & $8.43 \%$ & 3 & 4 & 0 \\
\hline Blood & 6 & $7.2 \%$ & 4 & 2 & 0 \\
\hline Bile fluid & 3 & $3.6 \%$ & 0 & 3 & 0 \\
\hline E.T secretion & 2 & $2.40 \%$ & 2 & 0 & 0 \\
\hline Total (percentage) & 83 & & $57(68.60 \%)$ & $24(29 \%)$ & $2(2.40 \%)$ \\
\hline
\end{tabular}

The highest incidence was found in elderly patients of age group 51-80 year age group, more common in males (70\%) than females (30\%) as shown in Table 2.

Table 2: Age group wise distribution.

\begin{tabular}{|lccc|}
\hline Age Group & Male & Female & Total \\
\hline $01-10$ & 0 & 1 & 1 \\
$11-20$ & 1 & 0 & 1 \\
$21-30$ & 2 & 4 & 6 \\
$31-40$ & 4 & 5 & 9 \\
$41-50$ & 5 & 3 & 8 \\
$51-60$ & 9 & 4 & 13 \\
$61-70$ & 16 & 8 & 24 \\
$71-80$ & 14 & 0 & 14 \\
$81-90$ & 7 & 0 & 7 \\
Total & 58 & 25 & 83 \\
Percentage & $70 \%$ & $30 \%$ & \\
\hline
\end{tabular}

The susceptibility pattern of various antimicrobials used in the Vitek 2 compact for each Citrobacter species isolated is given in Table 3 and Figure 1.

The susceptibility pattern observed in C. koseri is $100 \%$ in tigecycline and colistin. piperacillintazobactum (84\%), cefoperazone-sulbactum (94\%). Aminoglycosides such as amikacin and gentamycin showed good susceptibility of $96 \%$ and $98 \%$ each respectively. In carbapenems, ertapenem (91.2\%), imipenem (92\%), meropenem (89\%) also showed good susceptibility. In cephalosporins $3{ }^{\text {rd }}$ generation ceftriaxone showed $75.4 \%$ which is pretty low as compared to $4^{\text {th }}$ generation cefepime which showed $92 \%$. In fluoroquinolones, ciprofloxacin showed $87 \%$ susceptible.

Urine isolates of $C$. koseri showed nitrofurantoin (89.4\%), nalidixic acid (80.7\%) and trimethoprimsulfamethoxazole (96.4\%).

The susceptibility pattern observed in $C$. freundii it was slightly reduced with piperacillintazobactum (75\%), cefoperazone-sulbactum (83.3\%). Aminoglycosides such as amikacin and gentamycin showed $79 \%$ and $70.8 \%$ susceptible. In carbapenems, ertapenem (87.5\%), imipenem 
(91.6\%), meropenem (87.5\%) also showed good susceptibility. In cephalosporins $3^{\text {rd }}$ generation, ceftriaxone showed $62.5 \%$ and $4^{\text {th }}$ generation cefepime which showed $87.5 \%$ In fluoroquinolones, ciprofloxacin showed $75 \%$ susceptible. High end antimicrobials such as tigecycline and colistin showed excellent susceptibility of $100 \%$.

Table 3: Antibiotic susceptibility pattern of Citrobacter spp. isolates.

\begin{tabular}{|c|c|c|}
\hline \multirow{2}{*}{$\begin{array}{l}\text { Antimicrobial } \\
\text { agents }\end{array}$} & $\begin{array}{l}\text { Susceptible (C. } \\
\text { koseri) }\end{array}$ & $\begin{array}{l}\text { Susceptible (C. } \\
\text { freundii) }\end{array}$ \\
\hline & $\begin{array}{c}\text { Number(n)/ } \\
\text { Percentage(\%) }\end{array}$ & $\begin{array}{c}\text { Number(n)/ } \\
\text { Percentage (\%) }\end{array}$ \\
\hline Amikacin & $55(96.4)$ & $19(79.1)$ \\
\hline CIP-Ciprofloxacin & $50(87.7)$ & $18(75)$ \\
\hline CRO-Ceftriaxone & $43(75.43)$ & $15(62.5)$ \\
\hline CS-Colistin & $57(100)$ & $24(100)$ \\
\hline CXM-Cefuroxime & $30(52.63)$ & $14(58.3)$ \\
\hline ETP-Ertapenem & $52(91.2)$ & $21(87.5)$ \\
\hline FEP-Cefepime & $53(92)$ & $21(87.5)$ \\
\hline FT-Nitrofurantoin & $51(89.4)$ & 19(79) \\
\hline GM-Gentamicin & $56(98)$ & $17(70.8)$ \\
\hline IPM-Imipenem & $53(92)$ & $22(91.6)$ \\
\hline MEM-Meropenem & $51(89)$ & $21(87.5)$ \\
\hline NA-Nalidixic Acid & $46(80.7)$ & $15(62.5)$ \\
\hline $\begin{array}{l}\text { SFP- } \\
\text { Cefoperazone/ } \\
\text { Sulbactam }\end{array}$ & $54(94)$ & $20(83.3)$ \\
\hline $\begin{array}{l}\text { SXT- } \\
\text { Trimethoprim/ } \\
\text { Sulfamethoxazole }\end{array}$ & $55(96.4)$ & $18(75)$ \\
\hline TGC-Tigecycline & $57(100)$ & $24(100)$ \\
\hline $\begin{array}{l}\text { TZP-Piperacillin/ } \\
\text { Tazobactam }\end{array}$ & $48(84)$ & $18(75)$ \\
\hline
\end{tabular}

Again urine isolates in case of $C$. fruendii, showed nitrofurantoin $(79 \%)$, nalidixic acid $(62.5 \%)$ and trimethoprim-sulfamethoxazole $(75 \%)$. Tigecycline was not reported for urinary isolates.

Citrobacter spp inherit intrinsic resistance to certain antimicrobials. C. koseri, C. fruendii, and C. amalonaticus are intrinsically resistant to amipicillin whereas C. fruendii is intrinsically resistant to amoxicillin-clavulanate, cephalosporin I and II . Therefore, these antimicrobials were excluded while reporting for susceptibility [11].

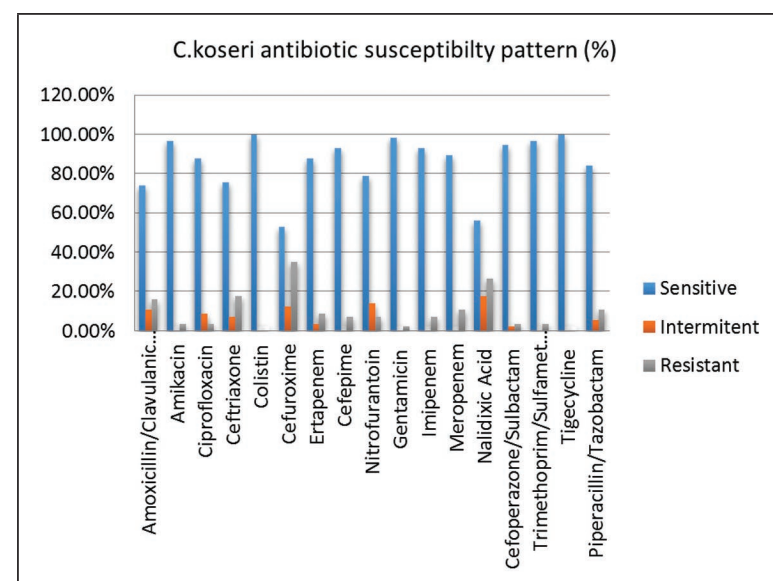

Figure 1: Graphical representation of C. koserii susceptibility.

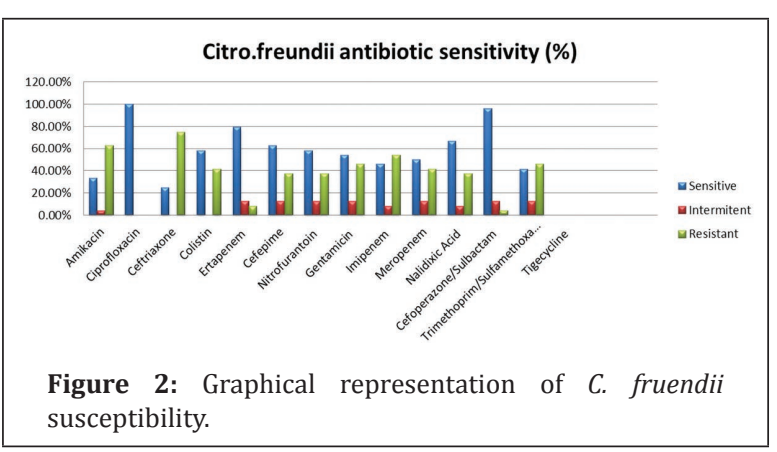

\section{Discussion}

The present study indicates the importance of Citrobacter species in various clinical samples as they are becoming an emerging pathogen with prevalence of $2.4 \%$ in 1 year. In the present study it has emerged as a potential uropathogen $56 \%$ followed by pus $21.6 \%$, sputum and blood with $8.4 \%$ and $7.2 \%$ respectively.

A study by Avinash et al. [2] mentioned the similar results of Citrobacter species, 150 (59.76\%) were from urine, $60(23.9 \%)$ from Pus, $8(7.17 \%)$ from blood, 16(6.37\%) from Sputum. Metri et al. [3] also concluded Citrobacter species as the third most common uropathogen isolated with prevalence of $15.7 \%$

In the present study C. koseri (68.60\%) is the most common species isolated from urine and pus followed 
by C. freundii (29\%) and C. amalonaticus $(2.40 \%)$ which is quite similar to the results of Dhanya et al. [12]. Similar results were also seen in study by Sami et al. [8] where $C$. koseri was the most abundant $(53.2 \%)$ followed by $C$. freundii $45(18.2 \%)$ and C.amalonaticus $24(9.75 \%)$ in urine samples. Metri et al. [13] found Citrobacter isolate accounting to 9.4\% with C. koseri $(72.4 \%)$ as predominant species in pus $(48.1 \%)$, followed by urine $(24.3 \%)$, sputum (20.3\%), body fluids $(05.2 \%)$, blood $(02.1 \%)$ which is pretty high as compared to the present study. However, on the contrary another study by Samsonis et al. [6] and Sneha et al. [1] mentioned C. freundii was more common $(71.8 \%)$ (49\%), followed by C. koseri $(23.1 \%),(28 \%)$ in urine and pus isolates respectively in their study.

The majority of age group affected in the present study is elderly age group of 51-80 years with male predominance which is similar to Metri et al. [13] and Samsonis et al. [6]. The reason of this could be because of the fact that these group of population have reduced immunity, having co-morbid diseases and more prone to hospitalisation.

In the present study the most common isolate identified is C. koseri $(68.60 \%)$ followed by $C$. freundii (29\%) and C. amalonaticus (2.40\%) therefore Antimicrobial susceptibility (AST) pattern was compared between $C$. koseri and $C$. freundii as C. amalonaticus isolated is too low to be commented upon.

In C. koseri, Beta lactam combinations showed good susceptibility piperacillin-tazobactum (84\%), cefoperazone-sulbactum (94\%) whereasin C. freundii it was slightly reduced with piperacillin-tazobactum (75\%), cefoperazone-sulbactum (83.3\%).

Aminoglycosides in case of $C$. koseri showed amikacin and gentamycin of good susceptibility of $96 \%$ and $98 \%$ each as compared to $79 \%$ and $70.8 \%$ in case of C. freundii.

In carbapenems, ertapenem $(91.2 \%, 87.5 \%)$, imipenem (92\%, 91\%), meropenem $(89 \%, 87.5 \%)$ showed good susceptibility in $C$. koseri and $C$. freundii respectively which is pretty similar to Metri et al. [3] and Avinash et al. [2].

In cephalosporins $3^{\text {rd }}$ generation ceftriaxone showed $75.4 \%$ and $62.5 \%$ which is pretty low as compared to $4^{\text {th }}$ generation cefepime which showed $92 \%$ and $87.5 \%$ in C. koseri and C. freundii respectively. In fluoroquinolones, ciprofloxacin showed $87 \%$ and $75 \%$ susceptibity in C. koseri and C. freundii respectively.

For urine isolates, nitrofurantoin $(89.4 \%, 79 \%)$, nalidixic acid $(80.7 \%, 62.5 \%)$ and trimethoprimsulfamethoxazole $(96.4 \%, 75 \%)$ in both C. koseri and C. freundii. High end antimicrobials such as tigecycline and colistin showed excellent susceptibility of $100 \%$ in both Citrobacter species isolated which correlates with study by Samsonis et al. [6].

Various studies observed the increased resistance in Citrobacter species in different parts of India but in the present study it shows considerable good susceptibility. This can be due to wide variation in specimen considered, hospital environment and hospital infection control policy.

\section{Conclusion}

The present study highlights that Citrobacter species are an emerging pathogen with prevalence of $2.4 \%$. It is the most common uropathogen followed by pus and sputum, however it is now shown to cause bacteraemia also. In this study, C. freundii was comparatively less susceptible as compared to $C$. koseri therefore more studies are required to keep the check on changing susceptibility pattern. The only limitation of the present study is isolation of $C$. amalonaticus is too less to comment on its susceptibility pattern. Hospital antibiotic policy should be implemented strictly so that emergence of such low virulent organism with emerging resistance can be controlled. Most importantly, strict adherence to infection prevention measures, implementation of antimicrobial stewardship programme and maintaining proper hand hygiene practices in tertiary care hospitals should be followed to prevent the transmission of this emerging pathogen.

\section{Conflicts of interest}

Authors declare no conflicts of interest.

\section{References}

[1] Mohan S, Agarwal J, Srivastava R, Singh M. Observations on Citrobacter species from a tertiary care health center with special reference to multi-drug resistance and presence of CTX-M gene. Indian J Pathol Microbiol. 2014; 57(3):439441. 
[2] John MS, Munilakshmi P, Deepa T. Significance of Citrobacter as an emerging nosocomial pathogen with special reference to it $\mathrm{s}$ antibiotic susceptibility pattern in a tertiary care hospital, Nellore, AP. India. Int J Curr Microbiol App Sci. 2015; 4(7):841-847.

[3] Metri BC, Jyothi P. Antibiotic sensitivity pattern of Citrobacter spp . Isolated from patients with urinary tract infections in tertiary care hospital in South India. Int J Pharm Pharmaceut Sci. 2015; 7(1):14-16.

[4] Metri BC, Jyothi P, Peerapur BV. Anti-microbial resistance profile of Citrobacter species in a tertiary care hospital of Southern India. Indian J Med Sci. 2011; 65(10):429-435.

[5] Liu L, Wang N, Wu AY, Lin C, Lee C, et al. Citrobacter freundii bacteremia: Risk factors of mortality and prevalence of resistance genes. J Microbiol Immunol Infect 2018; 51(4):565-572.

[6] Samonis G, Karageorgopoulos DE, Kofteridis DP, Matthaiou DK, Sidiropoulou V, et al. Citrobacter infections in a general hospital: characteristics and outcomes. Eur J Clin Microbiol Infect Dis. 2009; 28(1):61-68.

[7] Doran TI. The role of Citrobacter in clinical disease of children : Review. Clin Infect Dis. 1999; 28(2):384-394.

[8] Sami H, Sultan A, Rizvi M, Khan F, Ahmad S, et al. Citrobacteras a uropathogen , its prevalence and antibiotics susceptibility pattern. CHRISMED J Health Res. 2017; 4(1):23-26.

[9] Lavigne JP, Defez C, Bouziges N, Mahamat A, Sotto A. Clinical and molecular epidemiology of multidrug-resistant Citrobacter spp. infections in a French university hospital. Eur J Clin Microbiol Infect Dis. 2007; 26(6):439-441.

[10] Sb K, Thapa B, Mahat K. Nosocomial Citrobacter infection in neonatal intensive care unit in a hospital of Nepal. J Nepal Paed Soc. 2011; 31(2):105-109.

[11] Clinical and Laboratory Standards Institute (CLSI). Performance standards for antimicrobial susceptibilty testing. Twenty eighth informational supplement. CLSI Document M-100-28.Wayne, PA: 2018.

[12] Dhanya A, Sevitha Bhat. Clinicomicrobiological study of infections due to Citrobacter species. J Evol Med Dent Sci. 2015; 4(42):7327-7331.

[13] Metri BC, Jyothi P, Peerapur BV. Antibiotic resistance in Citrobacter spp. isolated from urinary tract infection. Urol Ann. 2013; 5(4):312-313. 